DOI: 10.20472/EFC.2018.010.008

KRZYSZTOF ECHAUST

Poznań University of Economics and Business, Poland

\title{
CONDITIONAL VAR USING GARCH-EVT APPROACH WITH OPTIMAL TAIL SELECTION
}

\begin{abstract}
:
Accurate risk prediction plays a key role in effective risk management process. A conditional GARCH-EVT approach combines Extreme Value Theory and GARCH methodology and it allows us to estimate Value at Risk with high accuracy. The approach requires to pre-specify a threshold indicating distribution tails. In this paper we use an optimal tail selection algorithm of Caeiro and Gomes (2016) to estimate out-of-sample VaR forecasts. Unlike other studies we update the optimal fraction of the tail for each rolling window of the data set. Results are presented for a long and a short position applying ten U.S. blue chips. The GARCH-EVT model enables us to estimate risk precisely. However, it is not possible to notice the improvement of $\mathrm{VaR}$ accuracy relative to conservative approach taking the 95th or 90th quantile of returns as a threshold.
\end{abstract}

\section{Keywords:}

Value-at-Risk, optimal tail selection, Extreme Value Theory, GARCH-EVT

JEL Classification: C22, C53 


\section{Introduction}

In recent years free movement of capital causes the prices of financial assets to become more dependent upon euphoria and panic investors. For all participants of financial markets it results in accepting a high risk of losses. The key issue in these circumstances is the correct measurement of an investment risk. The most common measure of the market risk is Value at Risk (VaR). VaR indicates how big is the maximum loss over target horizon such that there is a low, pre-specified probability that the actual loss will be larger. The main practical problem is the choice of appropriate method for measuring Value at Risk. The most popular and the simplest method is a historical simulation or variance-covariance method. When financial markets become violate and extreme returns appear, no method is able to measure risk appropriately.

Extreme Value Theory (EVT) provides a theoretical and practical foundation on statistical models describing extreme events. There is extensive literature that focus on EVT and discuss the tail behaviour of assets (Loretan and Phillips, 1994; Daníelsson and De Vries, 1997; McNeil, 1999; Neftci, 2000; Longin, 2000; Diebold, Schuermann and Stroughair, 2000; Jondeau and Rockinger, 2003; Fernandez, 2005; Gilli and Kellezi, 2006). EVT models deal with an iid time series, for that purpose McNeil and Frey (2000) build the combination of Extreme Value Theory and GARCH methodology called Conditional EVT or GARCH-EVT to capture the most important stylized facts with return data, as a volatility clustering and leptokurtosis, and quickly adapt to recent market movements. Usage of EVT in empirical research requires to pre-specify threshold which separates tails of distribution from its middle part. The choice of the threshold is ambiguous and affects the results of risk estimations.

This paper provides empirical study of conditional EVT. We analyse one day out-of-sample forecasts based on optimal tail selection algorithm of Caeiro and Gomes (2016). Unlike other studies we update the optimal fraction of the tail for each VaR forecast. In this way we can estimate the risk with the newest time horizon. We hypothesize that the optimal choice of tail fraction allows to improve the accuracy of VaR prediction. We analyze ten U.S. blue chips and present results for a long and a short position.

The remainder of the paper is organized as follows. Section 2 provides Peaks Over Threshold (POT) methodology. Section 3 describes tail selection problem in POT model. Section 4 explains the conditional VaR model. Section 5 describes backtesting tests. Section 6 presents empirical results for VaR forecasting. Section 7 concludes the study.

\section{$2 \quad$ Modelling tails using EVT}

Peaks Over Threshold, beside Block Maxima Model (BMM), is one of two key models of Extreme Value Theory. It allows to model the tail regions of the distribution instead of the entire sample. The Pickands-Balkema-de Haan Theorem (Balkema and de Haan, 1974) states, that for a high threshold $u$ the distribution function $F_{u}$ defined as:

$$
F_{u}(y)=P(X-u \leq x \mid X>u)=\frac{F(y+u)-F(u)}{1-F(u)} \text { for } 0<y<x_{0}-u,
$$

converges to the Generalized Pareto Distribution as $u \rightarrow x_{0}$, for the right endpoint $x_{0} \leq \infty$ of $F$. GPD is given by: 


$$
G_{\xi, \beta}(y)= \begin{cases}1-\left(1+\xi \frac{y}{\beta}\right)^{-\frac{1}{\xi}}, & \xi \neq 0, \\ 1-\exp \left(-\frac{y}{\beta}\right), & \xi=0,\end{cases}
$$

where $\beta>0, y \geq 0$ for $\xi \geq 0$ and $0 \leq y \leq-\beta / \xi$ for $\xi<0$. The distribution has only two parameters, $\beta$ is a scale parameter and $\xi$ is a shape parameter. Heavy tail distributions (i.e. stable, Student's $t$ ) have $\xi>0$ (Fréchet domain of attraction), whereas thin tail distributions like normal and log-normal have $\xi=0$ (Gumbel domain of attraction). Distributions with finite right endpoint have $\xi<0$ (Weibull domain of attraction). Rearranging (1)-(2) we obtain cumulative distribution function of returns:

$$
F(x)=(1-F(u)) G_{\xi, \beta}(y)(x-u)+F(u), \quad x>u .
$$

To obtain useful closed form of distribution (3) it is convenient to replace $F(u)$ by the empirical estimator of exceedance over a threshold. The estimator is of the form $\hat{F}(u)=1-N_{u} / n$, where $N_{u}$ is a number of returns that exceed the threshold $u$ and $n$ is number of returns. The estimator of cumulative distribution $F$ is then as follows:

$$
\hat{F}(x)=1-\frac{N_{u}}{n}\left(1+\xi \frac{(x-u)}{\beta}\right)^{-\frac{1}{\xi}} .
$$

VaR for a short position is given by the $x$ value in equation (4) and we receive:

$$
\operatorname{VaR}_{q}=u+\frac{\beta}{\xi}\left(\left(\frac{n}{N_{u}}(1-q)\right)^{-\xi}-1\right),
$$

where $q$ is the confidence level of VaR. In order to calculate VaR for a long position it is needed to carry out the calculations for minus returns.

\section{Tail selection}

The appropriate choice of threshold level $u$ is crucial in the estimation of Generalized Pareto Distribution parameters and the corresponding accuracy of Value-at-Risk. The standard practice is to adopt as low a threshold as possible but there is a trade-off between variance and bias. If a threshold is too low, the asymptotic basis of the model is violated leading to high bias. However, too high a threshold generates not enough excesses with which the model is estimated leading to high variance (Coles, 2001). Traditional ways for the choice of a threshold are based on graphical representations. An often used procedure is the analysis of a mean residual life plot which represents the mean of the excesses of the threshold $u$. This method is used by Aboura (2014), Omari, Mwita and Waititu (2017) to estimate VaR based on GARCH-EVT approach. Another very popular procedure to threshold selection is graphical representation of Hill (Hill, 1975), Pickands (Pickands, 1975) or Dekkers-Einmahl-de Haan estimators (Dekkers, Einmahl and Haan, 1989). The graphical based threshold choice procedures require to find stable regions in the graphs and thus they are highly subjective. Some authors chose threshold as a fixed quantile of the data set, especially when they use a moving window of observation to find out-of-sample VaR estimates. For instance Soltane, Karaa and Bellalah (2012) use a correction to Hill estimator and estimate GPD parameters in GARCH-EVT model keeping them constant for half a year of data in backtesting procedure. McNeil and Frey (2000), Karmakar and Shukla (2015), Bee at.al. (2016), Totić and Božović (2016), Li (2017) choose 90th quantile of the loss distribution as a threshold whereas Cifter (2011) uses 95th quantile.

There is extensive literature proposing the optimal choice of threshold, corresponding to the application of automated methods. The most common methods of adaptive choice of the threshold 
are based on the minimization of MSE's estimates (e.g. Hall, 1982; Hall and Welsh, 1985; Danielsson at al., 2001; Gomes and Oliveira, 2012). A recent overview of the topic can be found in (Danielsson at al., 2016).

In our empirical study we use an algorithm described in (Caeiro and Gomes, 2016). The algorithm searches for a stable region of the path stability (PS), i.e. the Hill plot of a tail index with respect to $k$. This is done in following steps:

Step 1. Given an observed returns $\left(r_{1}, \ldots, r_{n}\right)$, compute $T(k):=\hat{\xi}_{k, n}$ using Hill estimator for $k=$ $1, \ldots, n-1$.

Step 2. Obtain $j_{0}$ as a minimum value of $j$, a non-negative integer, such that the rounded values, to $j$ decimal places $\left(j=1\right.$ here), of the estimates $T(k)$ are distinct. Define $a_{k}^{(T)}(j)=$ round $(T(k), j), k=1, \ldots, n-1$, the rounded values of $T(k)$ to $j$ decimal places.

Step 3. Consider the set of $k$ values associated to equal consecutive values of $a_{k}^{(T)}\left(j_{0}\right)$ obtained in step 2. Set $k_{\min }^{(T)}$ and $k_{\max }^{(T)}$ the minimum and maximum values, respectively, of the set with the largest range. The largest run size is $l:=k_{\max }^{(T)}-k_{\min }^{(T)}$.

Step 4. Consider all those estimates, $T(k), k_{\text {min }}^{(T)} \leq k \leq k_{\text {max }}^{(T)}$, now with two additional decimal places, i.e. compute $T(k)=a_{k}^{(T)}\left(j_{0}+2\right)$. Obtain the mode of $T(k)$ and denote $\mathcal{K}_{T}$ the set of $k$ - values associated with this mode.

Step 5. Take $\hat{k}_{T}$ as the maximum value of $\mathcal{K}_{T}$.

Step 6. Compute $\hat{\xi}^{P S}=\hat{\xi}_{\hat{k}_{T}, n}$.

\section{GARCH-EVT model}

The GARCH-EVT model is a concept of McNeil and Frey (McNeil and Frey, 2000) to VaR modelling by extending the EVT framework to dependent data. It is a two-step procedure. First the GARCH model is fitted to returns using pseudo maximum likelihood approach. The standardized residuals of this model are extracted. If specified model is correct, the residuals of the model should be realizations of the unobserved iid noise variables. There are a lot of various variants of GARCH models. In this paper we use the most popular $\mathrm{GARCH}(1,1)$ (Bollerslev, 1986):

$$
\sigma_{t}^{2}=\omega+\alpha r_{t-1}^{2}+\beta \sigma_{t-1}^{2}
$$

where $\omega, \alpha, \beta>0, \alpha+\beta<1$. The high value of $\beta$ parameter implies that volatility is persistent and takes a long time to change. The high value of $\alpha$ means quick reaction of volatility to market movements. In the second stage a GPD parameters are fitted to standardized residuals $e_{t}$ of the GARCH model. VaR for short position (right tail) is calculated using the following expression:

$$
\operatorname{VaR}_{q}\left(r_{t}\right)=\sigma_{t}(1) \operatorname{VaR}_{q}\left(e_{t}\right),
$$

where $\sigma_{t}(1)$ is one step ahead prediction of next period scale parameter in GARCH type model and $\operatorname{VaR}_{q}\left(e_{t}\right)$ is calculated from (5) but for the standardized residuals $e_{t}$ of GARCH model.

\section{$5 \quad$ Backtesting tests}

Evaluation of the quality of VaR estimates can be made using backtesting procedure. The most popular backtest is the proportion of failures Kupiec's test (Kupiec, 1995). In this test the null hypothesis takes the following form: the observed number of exceedances is appropriate compared to the expected $\alpha$. The test statistic has the following form: 


$$
L R_{-} U C=2\left(\ln \left(\left(\frac{T_{1}}{T_{0}+T_{1}}\right)^{T_{1}}\left(1-\frac{T_{1}}{T_{0}+T_{1}}\right)^{T_{0}}\right)-\ln \left(\alpha^{T_{1}}(1-\alpha)^{T_{0}}\right)\right),
$$

where: $T_{1}$ - the number of VaR exceedances, $T_{0}$ - the number of unexceeded VaR.

With the true null hypothesis $L R \_U C$ statistic has the asymptotic chi-square distribution with one degree of freedom.

The Kupiec's test measures only the number of exceptions and ignores the time dynamics of exceedances. A good backtesting model should check also the independence property of exceedances. Christoffersen's test (Christoffersen, 1998) additionally checks independence of exceedances. If the model is accurate, then an exception today should not depend on whether or not an exception occurred on the previous day. The null hypothesis is: exceedances' percentage is consistent compared to the expected $\alpha$ and the exceedances are independent over time. The test statistic is of the form:

$$
\begin{gathered}
L R_{-} C C=2 \ln \left(\left(\frac{T_{01}}{T_{01}+T_{00}}\right)^{T_{01}}\left(1-\frac{T_{01}}{T_{01}+T_{00}}\right)^{T_{00}}\left(\frac{T_{11}}{T_{10}+T_{11}}\right)^{T_{11}}\left(1-\frac{T_{11}}{T_{10}+T_{11}}\right)^{T_{10}}\right)+ \\
-2 \ln \left(\alpha^{T_{01}+T_{11}}(1-\alpha)^{T_{00}+T_{10}}\right),
\end{gathered}
$$

where $T_{i j}$ - the number of days when condition $j$ occurred assuming that condition $i$ occurred on the previous day ( 1 if violation accurs, 0 if no violation accurs).

With the true null hypothesis $L R \_C C$ statistic has the asymptotic chi-square distribution with two degrees of freedom.

\section{Empirical findings}

To test the forecasting performance of the examined GARCH-EVT model with different choices of threshold we choose ten U.S. stocks as a base for analysis. They are constituents of the Dow Jones Index. Air Products \& Chemicals and Freeport-McMoRan are a members of the Basic Materials sector, Boeing and General Electric are from Industrial sector, EOG Recourses and Exxon Mobil Corporation belong to Oil\&Gas sector, Alphabet and Intel are members of the Technology sector and JPMorgan Chase \& Co. and Goldman Sachs Group are from Financial sector. The data consists of daily prices of the chosen assets from the beginning of 2005 up to the end of March 2018. It gives 3332 log-returns which are used in our calculations. Using rolling windows of size of 750 returns we update the estimates of parameters for each moving window.

According to the GARCH-EVT approach the tail fraction is estimated for standardized residuals of the $\mathrm{GARCH}$ model, not for returns. We used a $\operatorname{GARCH}(1,1)$ model with Gaussian innovations. Then we calculate tail fraction in three cases i.e. using optimal tail selection method of Caeiro and Gomes (2016), the 90th and 95th quantile of a data set. For all considered models, we allow the tail fraction and the model parameters to change over time. Figure 1 shows an example of tail behaviour for Alphabet Inc. It is easy to notice that that optimal tail is estimated to be much nearer to the 90th than the 95th quantile. For the remaining stocks (not presented here) we receive the similar results. Such choice of threshold guarantees enough data in tail to calculate VaR estimates at standard confidence levels. We tried also to use the optimal tail selection method of Danielsson et al. (2016). Unfortunately, this method generates optimal tails at a very high level and not enough data to calculate $\mathrm{VaR}$ at a high confidence level is available. 
Figure 1: Threshold in POT model for lower and upper tail. Black line - optimal tail, dark grey line -95 th quantile, light grey line -90 th quantile of standardized residuals of $\operatorname{GARCH}(1,1)$ model for Alphabet returns.

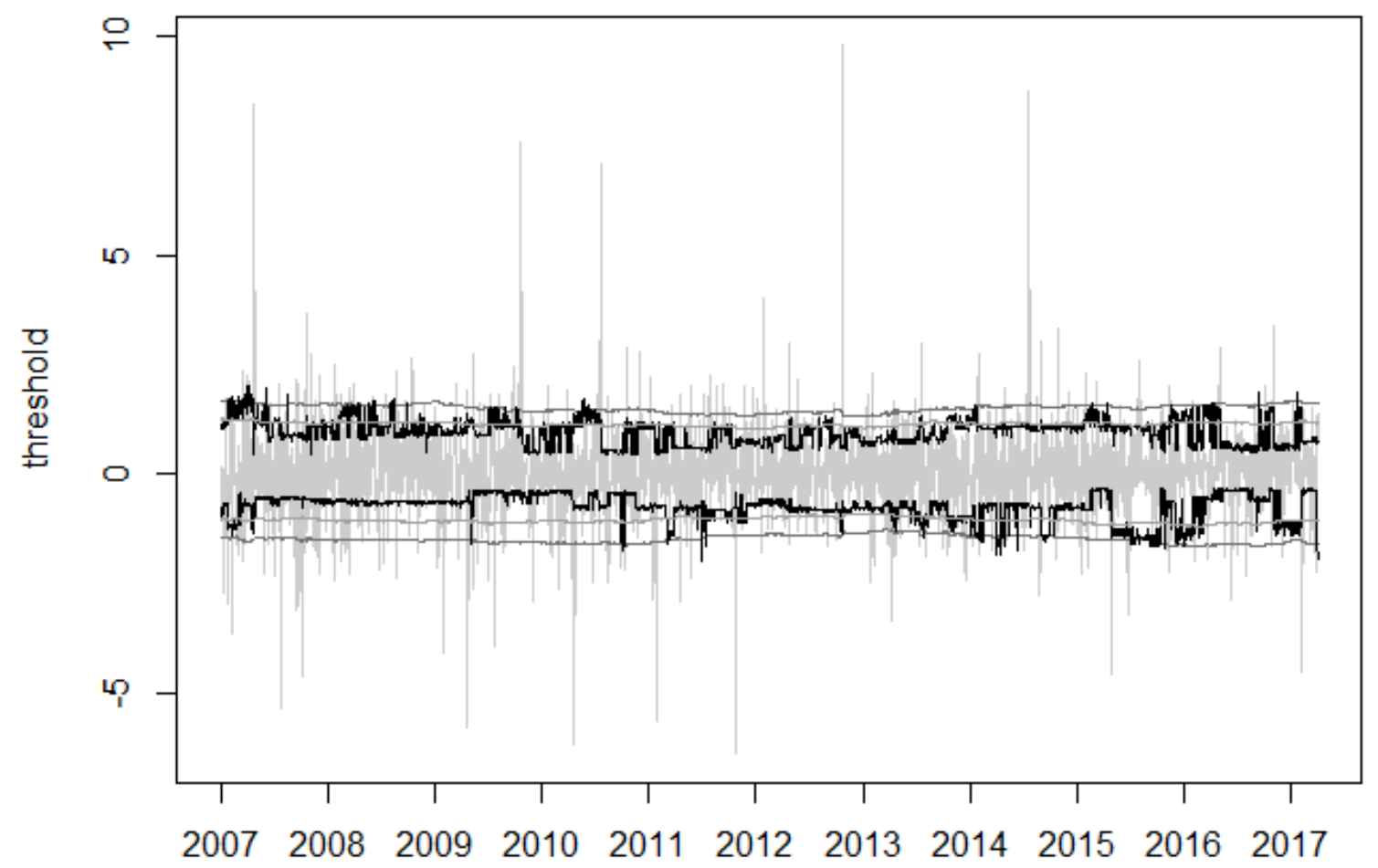

Source: own calculations

Having specified tails of distribution the next step in calculations is to estimate GDP parameters. The log-likelihood method is used which is described in Coles (2001). Figure 2 presents shape parameter behaviour for the example of Alphabet Inc. Since threshold based on optimal choice is near the 90th quantile we could expect shape parameters for these tail choices to behave similarly. Interestingly shape parameters are almost always positive. It means that residuals of the GARCH model do not follow Gaussian distribution and still have fat tails.

The final step of analysis is calculate VaR forecasts. We examine the out-of-sample $99.9 \%$, $99 \%$ and $95 \%$ VaR estimates for left and right tails of returns distribution. The Figure 3 presents $99 \%$ VaR estimates calculated from an optimal tail selection model for the Alphabet Inc. 
Figure 2: Shape parameter for upper tail (upper panel) and lower tail (lower panel) calculated for standardized residuals of GARCH $(1,1)$ model for Alphabet returns. Black line - optimal tail, dark grey line - 95th quantile, light grey line - 90th quantile of standardized residuals.
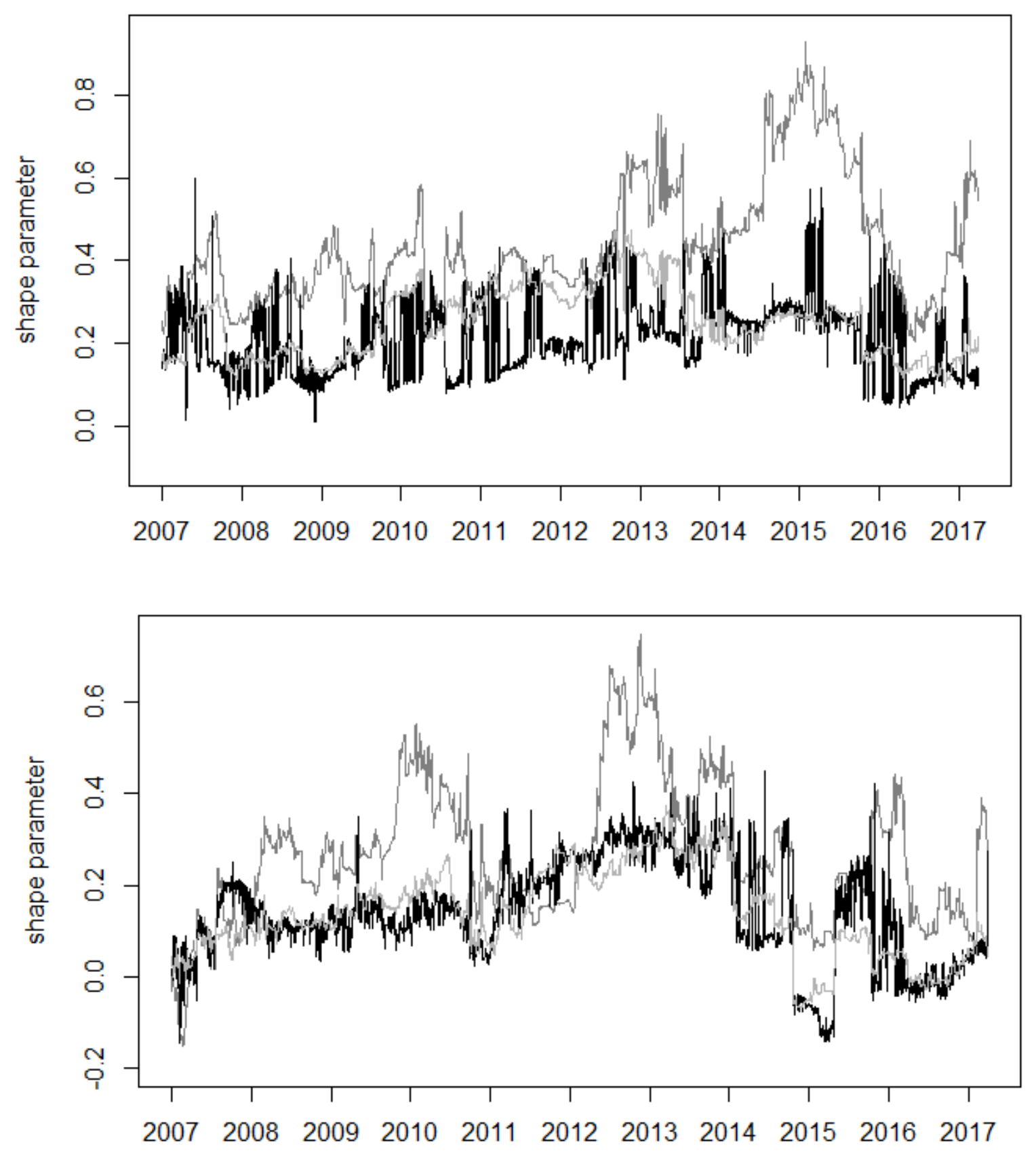

Source: own calculations 
Figure 3: Value at Risk at $99 \%$ confidence level for Alphabet returns

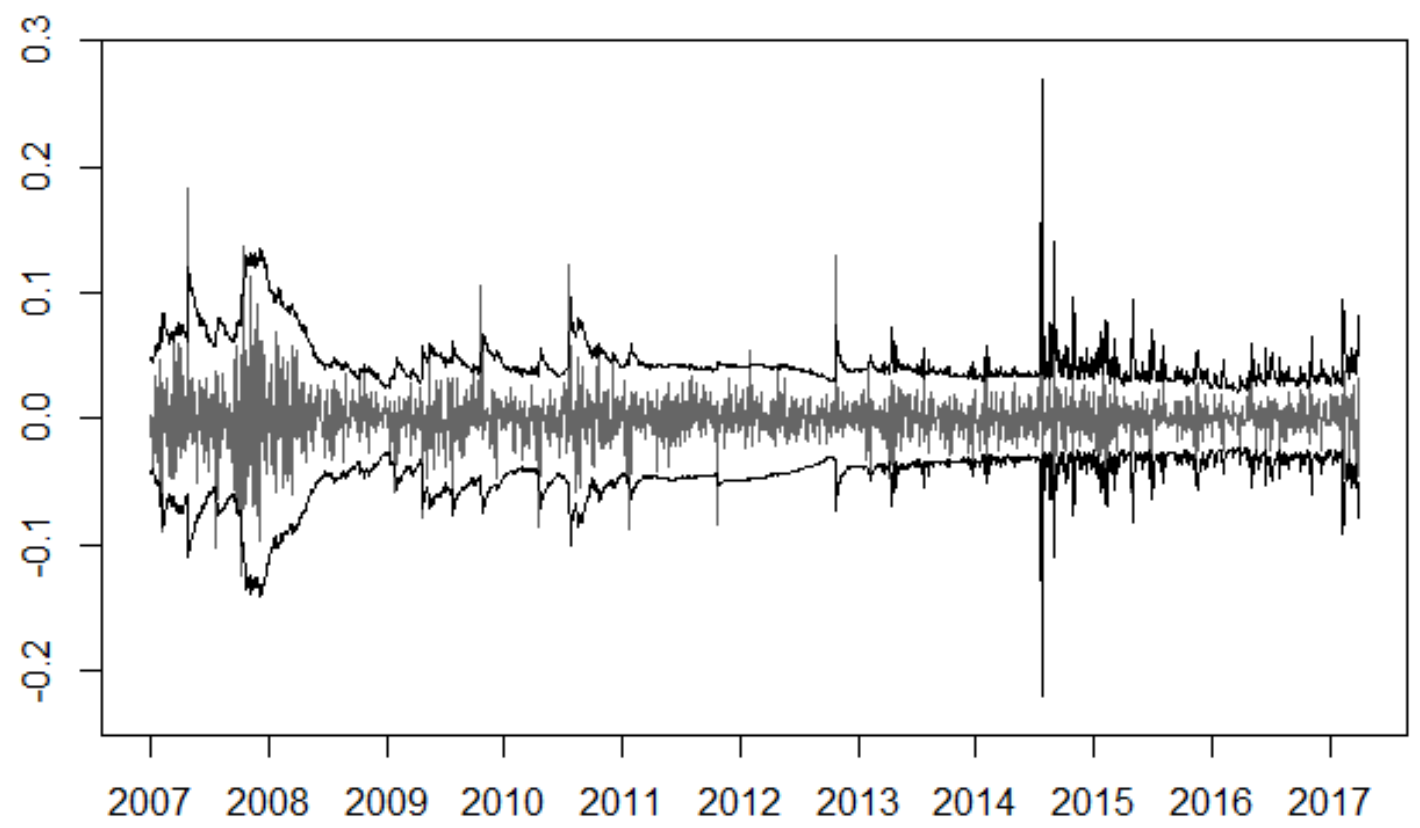

Source: own calculations

Backtesting results for all stocks and three choices of threshold are summarized in Tables 1-3. Assessing the quality of the estimated VaR, based on Kupiec's test, it can be concluded that the worst results are obtained for highly extreme VaR i.e. at $99.9 \%$ confidence level. The model is rejected for three stocks indicating a substential underestimation of the tail risk. For remaining confidence levels all VaR predictions are accurate. Note, that the same results we obtain regardless of which method of threshold choice is used. The results of Christoffersen's test are the same for the optimal choice of threshold and for the 90th quantile of the sample. The test indicates, that a sequence of violations is time dependent and VaR forecasts are inappropriate for FreeportMcMoRan Inc. at 99\% confidence level and for JPMorgan Chase \& Co. at 99\% and 95\% confidences. The three exceptions appear only for left tails of distribution (long position). For the right tails and for all stocks there is no significant reason to reject the null hypothesis of independence $\mathrm{VaR}$ exceedances over time. The worse tail performance is obtained for the model with $95 \%$ quantile as a threshold which generates an additional two exceptions for right tail and 99.9\% confidence level. Summing up, the conditional EVT model turns out to have a very good coverage of extreme quantiles of the loss distribution for most stocks. We verify that the results obtained are robust to the tail fraction choices made. 
Table 1: Backtesting for GARCH-EVT model with optimal tail selection. $E T$ - expected number of exceedances, $T_{1}-$ actual number of exceedances, $U C_{-} L-$ Kupiec's test, $C C_{-} L-$ Christoffersen's test.

\begin{tabular}{|c|c|c|c|c|c|c|c|}
\hline \multirow[b]{2}{*}{ Stock } & \multirow[b]{2}{*}{$E T$} & \multicolumn{3}{|c|}{ Long position, 99.9\% VaR } & \multicolumn{3}{|c|}{ Short position, $99.9 \%$ VaR } \\
\hline & & $T_{1}$ & $U C \_L$ & $C C \_L$ & $T_{1}$ & $U C \_L$ & $C C \_L$ \\
\hline apd & 2 & 5 & $1.775(0.183)$ & $1.794(0.408)$ & 5 & $1.775(0.183)$ & $1.794(0.408)$ \\
\hline ba & 2 & 3 & $0.064(0.800)$ & $0.071(0.965)$ & 5 & $1.775(0.183)$ & $1.794(0.408)$ \\
\hline ge & 2 & 5 & $1.775(0.183)$ & $1.794(0.408)$ & 3 & $0.064(0.800)$ & $0.071(0.965)$ \\
\hline eog & 2 & 4 & $0.666(0.414)$ & $0.679(0.712)$ & 7 & ${ }^{*} 5.134(0.023)$ & $5.172(0.075)$ \\
\hline fcx & 2 & 3 & $0.064(0.800)$ & $0.071(0.965)$ & 6 & $3.287(0.070)$ & $3.315(0.191)$ \\
\hline googl & 2 & 6 & $3.287(0.070)$ & $3.315(0.191)$ & 5 & $1.775(0.183)$ & $1.794(0.408)$ \\
\hline gs & 2 & 7 & *5.134 (0.023) & $5.172(0.075)$ & 3 & $0.064(0.800)$ & $0.071(0.965)$ \\
\hline intc & 2 & 4 & $0.667(0.414)$ & $0.679(0.712)$ & 7 & ${ }^{*} 5.134(0.023)$ & $5.142(0.075)$ \\
\hline jpm & 2 & 6 & $3.287(0.070)$ & $3.315(0.191)$ & 2 & $0.142(0.706)$ & $0.146(0.930)$ \\
\hline \multirow[t]{2}{*}{ xom } & 2 & 5 & $1.775(0.183)$ & $1.794(0.408)$ & 5 & $1.775(0.183)$ & $1.794(0.408)$ \\
\hline & & \multicolumn{3}{|c|}{ Long position, 99\% VaR } & \multicolumn{3}{|c|}{ Short position, 99\% VaR } \\
\hline Stock & $E T$ & $T_{1}$ & $U C \_L$ & $C C \_L$ & $T_{1}$ & $U C \_L$ & $C C \_L$ \\
\hline apd & 25 & 29 & $0.380(0.537)$ & $1.040(0.595)$ & 27 & $0.054(0.817)$ & $0.625(0.731)$ \\
\hline ba & 25 & 31 & $0.986(0.321)$ & $1.740(0.419)$ & 28 & $0.181(0.671)$ & $1.207(0.547)$ \\
\hline ge & 25 & 27 & $0.054(0.817)$ & $0.625(0.732)$ & 30 & $0.650(0.420)$ & $1.483(0.476)$ \\
\hline eog & 25 & 30 & $0.650(0.420)$ & $1.355(0.508)$ & 26 & $0.001(0.972)$ & $0.530(0.767)$ \\
\hline fcx & 25 & 25 & $0.027(0.870)$ & **10.253 (0.006) & 28 & $0.181(0.671)$ & $0.795(0.672)$ \\
\hline googl & 25 & 24 & $0.132(0.716)$ & $0.583(0.747)$ & 21 & $0.971(0.324)$ & $1.315(0.518)$ \\
\hline gs & 25 & 26 & $0.001(0.972)$ & $1.248(0.536)$ & 28 & $0.181(0.671)$ & $0.795(0.672)$ \\
\hline intc & 25 & 23 & $0.323(0.570)$ & $0.737(0.692)$ & 30 & $0.650(0.420)$ & $1.355(0.508)$ \\
\hline jpm & 25 & 26 & $0.001(0.972)$ & ${ }^{* \star 9} 9.763(0.008)$ & 30 & $0.650(0.420)$ & $1.355(0.508)$ \\
\hline \multirow[t]{2}{*}{ xom } & 25 & 29 & $0.380(0.537)$ & $1.040(0.595)$ & 32 & $1.388(0.239)$ & $2.192(0.334)$ \\
\hline & & \multicolumn{3}{|c|}{ Long position, 95\% VaR } & \multicolumn{3}{|c|}{ Short position, 95\% VaR } \\
\hline Stock & $E T$ & $T_{1}$ & $U C \_L$ & $C C \_L$ & $T_{1}$ & $U C \_L$ & $C C \_L$ \\
\hline apd & 129 & 136 & $0.381(0.537)$ & $0.537(0.647)$ & 115 & $1.68(0.195)$ & $1.684(0.431)$ \\
\hline ba & 129 & 133 & $0.123(0.726)$ & $2.522(0.284)$ & 127 & $0.036(0.849)$ & $0.539(0.764)$ \\
\hline ge & 129 & 142 & $1.316(0.251)$ & $2.626(0.269)$ & 124 & $0.215(0.643)$ & $1.009(0.604)$ \\
\hline eog & 129 & 123 & $0.308(0.579)$ & $2.155(0.341)$ & 120 & $0.691(0.406)$ & $0.725(0.696)$ \\
\hline fcx & 129 & 129 & $0,000(0.993)$ & $0.388(0.824)$ & 136 & $0.382(0.537)$ & $3.697(0.157)$ \\
\hline googl & 129 & 131 & $0.029(0.864)$ & $0.869(0.648)$ & 116 & $1.446(0.229)$ & $1.456(0.483)$ \\
\hline gs & 129 & 132 & $0.068(0.794)$ & $0.164(0.921)$ & 125 & $0.138(0.710)$ & $0.353(0.838)$ \\
\hline intc & 129 & 114 & $0.944(0.331)$ & $1.220(0.543)$ & 133 & $0.123(0.726)$ & $1.607(0.448)$ \\
\hline jpm & 129 & 113 & $2.202(0.138)$ & ${ }^{* *} 14.819(0.006)$ & 110 & $3.125(0.077)$ & $3.240(0.198)$ \\
\hline xom & 129 & 139 & $0.780(0.377)$ & $2.414(0.299)$ & 129 & $0000(0.993)$ & $0.280(0.986)$ \\
\hline
\end{tabular}

Note: statistical significance level ${ }^{*}$ for $\mathrm{p}<0.05,{ }^{* *}$ for $\mathrm{p}<0.01$ ( $p$-values in parentheses).

Source: own calculations 
Table 2: Backtesting for GARCH-EVT model with 95th quantile for tail selection. ET expected number of exceedances, $T_{1}$ - actual number of exceedances, UC_L - Kupiec's test, CC_L - Christoffersen's test.

\begin{tabular}{|c|c|c|c|c|c|c|c|}
\hline \multirow[b]{2}{*}{ Stock } & \multirow[b]{2}{*}{$E T$} & \multicolumn{3}{|c|}{ Long position, 99.9\% VaR } & \multicolumn{3}{|c|}{ Short position, $99.9 \%$ VaR } \\
\hline & & $T_{1}$ & $U C \_L$ & $C C \_L$ & $T_{1}$ & $U C \_L$ & $C C \_L$ \\
\hline apd & 2 & 5 & $1.775(0.183)$ & $1.794(0.408)$ & 4 & $0.666(0.414)$ & $0.679(0.712)$ \\
\hline ba & 2 & 6 & $3.287(0.070)$ & $3.315(0.191)$ & 4 & $0.666(0.414)$ & $0.679(0.712)$ \\
\hline ge & 2 & 6 & $3.287(0.070)$ & $3.315(0.191)$ & 3 & $0.064(0.800)$ & $0.071(0.965)$ \\
\hline eog & 2 & 6 & $3.287(0.070)$ & $3.315(0.191)$ & 8 & $\star * 7.269(0.007)$ & *7.319 (0.026) \\
\hline fcx & 2 & 4 & $0.666(0.414)$ & $0.679(0.712)$ & 6 & $3.287(0.070)$ & $3.315(0.191)$ \\
\hline googl & 2 & 5 & $1.775(0.183)$ & $1.794(0.408)$ & 5 & $1.775(0.183)$ & $1.794(0.408)$ \\
\hline gs & 2 & 7 & *5.134 (0.023) & $5.172(0.075)$ & 3 & $0.064(0.800)$ & $0.071(0.965)$ \\
\hline intc & 2 & 5 & $1.775(0.183)$ & $1.794(0.408)$ & 8 & $\star * 7.269(0.007)$ & *7.319 (0.026) \\
\hline jpm & 2 & 6 & $3.287(0.070)$ & $3.315(0.191)$ & 5 & $1.775(0.183)$ & $1.794(0.408)$ \\
\hline \multirow[t]{2}{*}{ xom } & 2 & 5 & $1.775(0.183)$ & $1.794(0.408)$ & 5 & $1.775(0.183)$ & $1.794(0.408)$ \\
\hline & & \multicolumn{3}{|c|}{ Long position, 99\% VaR } & \multicolumn{3}{|c|}{ Short position, 99\% VaR } \\
\hline Stock & $E T$ & $T_{1}$ & $U C \_L$ & $C C \_L$ & $T_{1}$ & $U C \_L$ & $C C \_L$ \\
\hline apd & 25 & 32 & $1.388(0.239)$ & $2.192(0.334)$ & 26 & $0.001(0.972)$ & $0.767(0.536)$ \\
\hline ba & 25 & 30 & $0.650(0.420)$ & $1.355(0.508)$ & 28 & $0.181(0.671)$ & $1.207(0.547)$ \\
\hline ge & 25 & 31 & $0.986(0.321)$ & $1.733(0.420)$ & 29 & $0.380(0.537)$ & $1.040(0.595)$ \\
\hline eog & 25 & 30 & $0.650(0.420)$ & $1.355(0.508)$ & 28 & $0.181(0.671)$ & $0.795(0.672)$ \\
\hline fcx & 25 & 27 & $0.054(0.817)$ & ${ }^{* * 9.371(0.009)}$ & 29 & $0.380(0.537)$ & $1.040(0.595)$ \\
\hline googl & 25 & 26 & $0.001(0.972)$ & $0.530(0.767)$ & 23 & $0.323(0.570)$ & $0.737(0.692)$ \\
\hline gs & 25 & 29 & $0.380(0.537)$ & $1.307(0.520)$ & 27 & $0.054(0.817)$ & $0.625(0.731)$ \\
\hline intc & 25 & 25 & $0.027(0.870)$ & $0.516(0.773)$ & 32 & $1.388(0.239)$ & $2.192(0.334)$ \\
\hline jpm & 25 & 29 & $0.380(0.537)$ & *8.870 (0.011) & 27 & $0.054(0.817)$ & $0.625(0.731)$ \\
\hline \multirow[t]{2}{*}{ xom } & 25 & 30 & $0.650(0.420)$ & $1.483(0.476)$ & 28 & $0.181(0.671)$ & $0.795(0.672)$ \\
\hline & & \multicolumn{3}{|c|}{ Long position, 95\% VaR } & \multicolumn{3}{|c|}{ Short position, 95\% VaR } \\
\hline Stock & $E T$ & $T_{1}$ & $U C \_L$ & $C C \_L$ & $T_{1}$ & $U C \_L$ & $C C \_L$ \\
\hline apd & 129 & 133 & $0.123(0.726)$ & $0.810(0.667)$ & 119 & $0.853(0.356)$ & $0.902(0.637)$ \\
\hline ba & 129 & 129 & $0.000(0.993)$ & $2.999(0.223)$ & 126 & $0.079(0.779)$ & $0.645(0.724)$ \\
\hline ge & 129 & 142 & $1.316(0.251)$ & $2.626(0.269)$ & 125 & $0.138(0.710)$ & $0.353(0.838)$ \\
\hline eog & 129 & 125 & $0.138(0.710)$ & $1.003(0.606)$ & 117 & $1.231(0.267)$ & $1.250(0.535)$ \\
\hline fcx & 129 & 130 & $0.007(0.935)$ & $0.929(0.629)$ & 132 & $0.068(0.794)$ & $2.932(0.231)$ \\
\hline googl & 129 & 128 & $0.010(0.921)$ & $0.082(0.960)$ & 115 & $1.680(0.195)$ & $1.684(0.431)$ \\
\hline gs & 129 & 134 & $0.193(0.660)$ & $0.194(0.908)$ & 124 & $0.215(0.643)$ & $0.393(0.821)$ \\
\hline intc & 129 & 137 & $0.499(0.480)$ & $1.533(0.465)$ & 136 & $0.382(0.537)$ & $3.697(0.157)$ \\
\hline jpm & 129 & 115 & $1.680(0.195)$ & $* * 13.579(0.001)$ & 117 & $1.231(0.267)$ & $1.612(0.447)$ \\
\hline xom & 129 & 125 & $0.138(0.710)$ & $2.555(0.279)$ & 133 & $0.123(0.726)$ & $0.232(0.891)$ \\
\hline
\end{tabular}

Note: statistical significance level ${ }^{*}$ for $\mathrm{p}<0.05,{ }^{* *}$ for $\mathrm{p}<0.01$ ( $p$-values in parentheses).

Source: own calculations 
Table 3: Backtesting for GARCH-EVT model with 90th quantile for tail selection. ET expected number of exceedances, $T_{1}$ - actual number of exceedances, UC_L - Kupiec's test, CC_L - Christoffersen's test.

\begin{tabular}{|c|c|c|c|c|c|c|c|}
\hline \multirow[b]{2}{*}{ Stock } & \multirow[b]{2}{*}{$E T$} & \multicolumn{3}{|c|}{ Long position, 99.9\% VaR } & \multicolumn{3}{|c|}{ Short position, 99.9\% VaR } \\
\hline & & $T_{1}$ & $U C \_L$ & $C C \_L$ & $T_{1}$ & $U C \_L$ & $C C \_L$ \\
\hline apd & 2 & 5 & $1.775(0.183)$ & $1.794(0.408)$ & 4 & $0.666(0.414)$ & $0.679(0.712)$ \\
\hline ba & 2 & 4 & $0.666(0.414)$ & $0.679(0.712)$ & 5 & $1.775(0.183)$ & $1.794(0.408)$ \\
\hline ge & 2 & 4 & $0.666(0.414)$ & $0.679(0.712)$ & 7 & *5.134 (0.023) & $5.172(0.075)$ \\
\hline eog & 2 & 4 & $0.666(0.414)$ & $0.679(0.712)$ & 6 & $3.287(0.070)$ & $3.315(0.191)$ \\
\hline fcx & 2 & 6 & $3.287(0.070)$ & $3.315(0.191)$ & 3 & $0.064(0.800)$ & $0.071(0.965)$ \\
\hline googl & 2 & 7 & *5.134 (0.023) & $5.172(0.075)$ & 5 & $1.775(0.183)$ & $1.794(0.408)$ \\
\hline gs & 2 & 7 & *5.134 (0.023) & $5.172(0.075)$ & 3 & $0.064(0.800)$ & $0.071(0.965)$ \\
\hline intc & 2 & 5 & $1.775(0.183)$ & $1.794(0.408)$ & 6 & $3.287(0.070)$ & $3.315(0.191)$ \\
\hline jpm & 2 & 6 & $3.287(0.070)$ & $3.315(0.191)$ & 3 & $0.064(0.800)$ & $0.071(0.965)$ \\
\hline \multirow[t]{2}{*}{ xom } & 2 & 5 & $1.775(0.183)$ & $1.794(0.408)$ & 4 & $0.666(0.414)$ & $0.679(0.712)$ \\
\hline & & \multicolumn{3}{|c|}{ Long position, 99\% VaR } & \multicolumn{3}{|c|}{ Short position, 99\% VaR } \\
\hline Stock & $E T$ & $T_{1}$ & $U C \_L$ & $C C \_L$ & $T_{1}$ & $U C \_L$ & $C C \_L$ \\
\hline apd & 25 & 28 & $0.181(0.671)$ & $0.795(0.672)$ & 26 & $0.001(0.972)$ & $0.530(0.767)$ \\
\hline ba & 25 & 31 & $0.986(0.321)$ & $1.740(0.419)$ & 28 & $0.181(0.671)$ & $1.207(0.547)$ \\
\hline ge & 25 & 30 & $0.650(0.420)$ & $1.355(0.508)$ & 27 & $0.054(0.817)$ & $0.625(0.731)$ \\
\hline eog & 25 & 25 & $0.027(0.870)$ & **10.253 (0.006) & 28 & $0.181(0.671)$ & $0.795(0.672)$ \\
\hline fcx & 25 & 27 & $0.054(0.817)$ & $0.625(0.732)$ & 28 & $0.181(0.671)$ & $1.207(0.547)$ \\
\hline googl & 25 & 25 & $0.027(0.870)$ & $0.516(0.773)$ & 20 & $1.437(0.231)$ & $1.749(0.417)$ \\
\hline gs & 25 & 25 & $0.027(0.870)$ & $1.394(0.498)$ & 29 & $0.380(0.537)$ & $1.040(0.595)$ \\
\hline intc & 25 & 22 & $0.601(0.438)$ & $0.979(0.613)$ & 29 & $0.380(0.537)$ & $1.040(0.595)$ \\
\hline jpm & 25 & 27 & $0.054(0.817)$ & ${ }^{* * 9.371(0.009)}$ & 29 & $0.380(0.537)$ & $1.040(0.595)$ \\
\hline \multirow[t]{2}{*}{ xom } & 25 & 29 & $0.380(0.537)$ & $1.307(0.520)$ & 33 & $1.854(0.173)$ & $2.709(0.258)$ \\
\hline & & \multicolumn{3}{|c|}{ Long position, 95\% VaR } & \multicolumn{3}{|c|}{ Short position, 95\% VaR } \\
\hline Stock & $E T$ & $T_{1}$ & $U C \_L$ & $C C \_L$ & $T_{1}$ & $U C \_L$ & $C C \_L$ \\
\hline apd & 129 & 134 & $0.193(0.660)$ & $0.810(0.667)$ & 120 & $0.691(0.406)$ & $0.759(0.684)$ \\
\hline ba & 129 & 131 & $0.029(0.864)$ & $2.719(0.257)$ & 130 & $0.007(0.935)$ & $0.342(0.843)$ \\
\hline ge & 129 & 123 & $0.308(0.579)$ & $2.155(0.341)$ & 120 & $0.691(0.406)$ & $0.725(0.696)$ \\
\hline eog & 129 & 131 & $0.029(0.864)$ & $0.050(0.976)$ & 135 & $0.271(0.597)$ & $3.463(0.177)$ \\
\hline fcx & 129 & 143 & $1.525(0.217)$ & $2.735(0.255)$ & 122 & $0.418(0.518)$ & $0.536(0.765)$ \\
\hline googl & 129 & 131 & $0.029(0.864)$ & $0.869(0.648)$ & 119 & $0.853(0.356)$ & $0.902(0.637)$ \\
\hline gs & 129 & 133 & $0.123(0.726)$ & $0.246(0.884)$ & 122 & $0.418(0.518)$ & $0.536(0.765)$ \\
\hline intc & 129 & 136 & $0.382(0.537)$ & $0.869(0.648)$ & 134 & $0.193(0.660)$ & $1.772(0.412)$ \\
\hline jpm & 129 & 116 & $1.447(0.229)$ & ${ }^{* *} 15.400(0.000)$ & 109 & $3.470(0.062)$ & 3.560 (0.169) \\
\hline xom & 129 & 131 & $0.029(0.864)$ & $1.677(0.432)$ & 131 & $0.029(0.864)$ & $0.056(0.972)$ \\
\hline
\end{tabular}

Note: statistical significance level ${ }^{*}$ for $\mathrm{p}<0.05,{ }^{* *}$ for $\mathrm{p}<0.01$ ( $p$-values in parentheses).

Source: own calculations 


\section{Conclusions}

The selection of an appropriate tail fraction of distribution in one of the main problems in usage of Peaks Over Threshold method in practice. POT offers methodology for extreme risk measurement but it is highly dependent on the threshold which indicates the tail of distribution. The main contribution of this paper is to conduct a comparative study of predictive ability of VaR estimates when each estimate is made with optimal choice of distribution tail. The GARCH-EVT model is used because it is able to capture stylized facts, like heteroscedasticity and fat tails, about empirical returns. We compared the results to VaR estimates with fixed quantile (95th, 99th and 99.9th) of distribution as a threshold. Such choices of threshold are seen as standard in conditional EVT approach. GARCH-EVT model performs pretty well in estimating risk for all choices of threshold. Backtesting procedures indicate that no matter how the choice of the tail is taken, approximately the same accuracy of $\mathrm{VaR}$ prediction we receive. This conclusion presents a real dilemma to the importance of appropriate choice of a threshold. We find that the optimal tail selection does not improve accuracy of the VaR prediction relative to standard methods of choice of tail fraction and hence our research hypothesis is not confirmed. From a practical point of view the result means that investors may calculate conditional VaR using GARCH-EVT approach without reflection on distribution tails. Taking 5th percentile of the sample as a threshold value is sufficient to obtain accurate estimation of $\mathrm{VaR}$.

\section{References}

ABOURA, S. (2014). When the U.S. Stock Market Becomes Extreme? Risks, 2 (2), 211-225.

BALKEMA, A. A. and de HAAN, L. (1974). Residual Life Time at Great Age. The Annals of Probability, 2 , 792-804.

BEE, M., DUPUIS, D. J. and TRAPIN, L. (2016). Realizing the extremes: Estimation of tail-risk measures from a high-frequency perspective. Journal of Empirical Finance, 36, 86-99.

BOLLERSLEV, T. (1986). Generalized autoregressive conditional heteroskedasticity. Journal of Econometrics, 31, 307-327.

CAEIRO, J. and GOMES, M.I. (2016) Threshold selection in extreme value analysis. Extreme Value Modeling and Risk Analysis: Methods and Applications, 69-86.

CHRISTOFFERSEN, P. F. (1998). Evaluating Interval Forecasts. International Economic Review, 39, 841 862.

CIFTER, A. (2011). Value-at-risk estimation with wavelet-based extreme value theory: Evidence from emerging markets. Physica A: Statistical Mechanics and Its Applications, 390, 2356-2367.

COLES, S. G. (2001). An introduction to Statistical Modeling of Extreme Values. Springer Series in Statistics.

DANÍELSSON, J. and De VRIES, C. G. (1997). Tail index and quantile estimation with very high frequency data. In Journal of Empirical Finance, 4, 241-257.

DANIELSSON, J., ERGUN, L.M. and de HAAN, L. and de VRIES, C.G. (2016). Tail Index Estimation: Quantile Driven Threshold Selection. SRC Working paper.

DANIELSSON, J., HAAN, L., PENG, L. and VRIES, C.G. (2001) Using a bootstrap method to choose the sample fraction in tail index estimation. Journal of Multivariate Analysis, 76, 226-248.

DEKKERS, A. L. M., EINMAHL, J. H. J., and HAAN, L. De. (1989). A Moment Estimator for the Index of an 
Extreme-Value Distribution. The Annals of Statistics, 17(4), 1833-1855.

DIEBOLD, F. X., SCHUERMANN, T., and STROUGHAIR, J. D. (2000). Pitfalls and Opportunities in the Use of Extreme Value Theory in Risk Management. The Journal of Risk Finance, 1, 30-36.

FERNANDEZ, V. (2005). Risk management under extreme events. International Review of Financial Analysis, 14(2), 113-148.

GILLI, M. and KELLEZI, E. (2006). An Application of Extreme Value Theory for Measuring Financial Risk. Computational Economics, 27, 207-228.

GOMES, M. I. and OLIVEIRA, O. (2001), The bootstrap methodology in statistics of extremes - choice of the optimal sample fraction," Extremes, 4, 331-358.

HALL, P. (1982), On some simple estimates of an exponent of regular variation," Journal of the Royal Statistical Society. Series B (Methodological), 37-42.

HALL, P. (1990) Using the Bootstrap to Estimate Mean Squared Error and Select Smoothing Parameter in Nonparametric Problems. Journal of Multivariate Analysis, 32, 177-203.

HALL, P. and WELSH, A. (1985), Adaptive estimates of parameters of regular variation," The Annals of Statistics, 13, 331-341.

HILL, B. M. (1975). A Simple General Approach to Inference About the Tail of a Distribution. The Annals of Statistics, 3, 1163-1174.

JONDEAU, E. and ROCKINGER, M. (2003). Testing for differences in the tails of stock-market returns. Journal of Empirical Finance, 10, 559-581.

KARMAKAR, M., and SHUKLA, G. K. (2015). Managing extreme risk in some major stock markets: An extreme value approach. International Review of Economics \& Finance, 35, 1-25.

KUPIEC, P. H. (1995). Techniques for Verifying the Accuracy of Risk Measurement Models. The Journal of Derivatives, 3, 73-84.

LI, L. (2017): A Comparative Study of GARCH and EVT Model in Modeling Value-at-Risk. Journal of Applied Business and Economics, Vol. 19, No. 7 : pp. 27-48.

LONGIN, M. (2000). From value at risk to stress testing: The extreme value approach. Journal of Banking \& Finance, Vol. 24, No. 7, pp. 1097-1130.

LORETAN, M. and PHILLIPS, P. C. B. (1994). Testing the covariance stationarity of heavy-tailed time series: An overview of the theory with applications to several financial datasets. Journal of Empirical Finance, $1,211-248$.

MCNEIL, A. J. and FREY, R. (2000). Estimation of tail-related risk measures for heteroscedastic financial time series: An extreme value approach. Journal of Empirical Finance, 7, 271-300.

MCNEIL, A. J. (1999). Extreme Value Theory for Risk Managers A General Introduction to Extreme Risk. Internal Modelling and CAD II.

NEFTCI, S. N. (2000). Value at Risk Calculations, Extreme Events, and Tail Estimation. The Journal of Derivatives, 7, 23-38.

PICKANDS, J. . (1975). Statistical inference using extreme order statistics. Annals of Statistics, 3, 119-131.

OMARI, C. , MWITA, P. and WAITITU, A. (2017). Using Conditional Extreme Value Theory to Estimate Valueat-Risk for Daily Currency Exchange Rates. Journal of Mathematical Finance, 7, 846-870.

SOLTANE, H.B., KARAA, A. and BELLALAH M. (2012). Conditional VaR using GARCH-EVT approach: Forecasting Volatility in Tunisian Financial Market, Journal of Computations and Modelling, vol.2, no.2, 2012, 95-115. 
TOTIĆ, S. and BOŽOVIĆ, M. (2016). Tail risk in emerging markets of Southeastern Europe. Applied Economics, 48(19), 1785-1798. 